\title{
Stoffwechsel der weißen Blutzellen
}

\author{
Von Otto Warburg, Karlfried Gawehn und August-Wilhelm Geissler \\ Aus dem Max-Planck-Institut für Zellphysiologie, Berlin-Dahlem \\ (Z. Naturforschg. 13 b, 515-516 [1958] ; eingegangen am 21. Juni 1958)
}

\begin{abstract}
Der „Krebsstoffwechsel“ der normalen weißen Blutzellen, der vielfach, in der letzten Zeit z. B. von W. Remmele und F. Seelich ${ }^{1}$, gefunden wurde, ist ein Artefakt infolge mechanischer und chemischer Schädigungen.
\end{abstract}

Da in den weißen Blutzellen, ähnlich wie in anderen normalen Körperzellen ${ }^{2}$, aerobe Gärung durch Entkopplung der Atmung erzeugt wird, wenn man die Zellen aus Blutplasma in Salzlösungen bringt, so darf bei der Manometrie der weißen Blutzellen nur Plasma (das mit 2 bis $5 \mathrm{Vol} . \% \mathrm{CO}_{2}$ Luft gesättigt ist) verwendet werden. Es ist ferner bei der Gewinnung der weißen Blutzellen für manometrische Versuche ein Zusammenzentrifugieren zu vermeiden, da die empfindlichen Zellen dabei mechanisch geschädigt werden. Es ist außerdem zu beachten, daß die weißen Blutzellen Abkühlung auf $0^{\circ}$, wie manche andere Körperzellen, ohne Schädigung nicht vertragen. Beachtet man dies, so wird man, wie die folgenden Versuche zeigen, niemals „Krebsstoffwechsel", sondern immer einen rein aeroben Stoffwechsel der normalen menschlichen weißen Blutzellen finden.

Menschenblut fließt direkt aus der Kanüle in ein Drittel des Blutvolumens einer Salzlösung, die 0,7 g $\mathrm{NaCl}$ und $1,1 \mathrm{~g} \mathrm{Na}$-citrat $\cdot 5 \mathrm{H}_{2} \mathrm{O}$ enthält und $0,9 \%$ $\mathrm{NaCl}$ etwa isoton ist. Wird dann einige Min. bei $400 \mathrm{~g}$ zentrifugiert, so senken sich die roten Blutzellen schneller als die weißen, und man kann eine überstehende Flüssigkeit abheben, die fast nur weiße Blutzellen und Blutplättchen enthält. Wir pipettieren sie direkt in die Manometriegefäße und messen nach Zusatz von Glucose und Lactat ihren Stoffwechsel bei $38^{\circ}$ und Sättigung mit $5 \% \mathrm{CO}_{2}$-Luft, wie früher beschrieben ${ }^{2}$.

Abb. 1. ist die graphische Darstellung eines Versuches mit einigen mg Zellen. Wie man sieht, sind die manometrischen Ausschläge mehr als ausreichend und im Lauf einiger Stdn. nahezu konstant. Die Druckänderungen sind für die beiden Gefäße der 2-Gefäß-Methode negativ, so wie für normale Körperzellen; während Krebszellen unter sonst gleichen

1 W. Remmele, Acta Haematol. 23, 103 [1955]; F. Seelich, Z. Krebsforsch. 62, 1 [1957].

2 O. Warburg, K. Gawehn u. A. W. Geissler, Z. Naturforschg. 11 b, 657 [1956]; 12 b, 115 [1957].
Bedingungen in denselben Gefäßen positive Drucke ${ }^{3}$ geben. Die beiden gestrichelten Linien im positiven Gebiet der Abb. 1 zeigen die manometrischen Ausschläge, die die weißen Blutzellen geben würden, wenn ihr Stoffwechsel „Krebsstoffwechsel“ wäre.

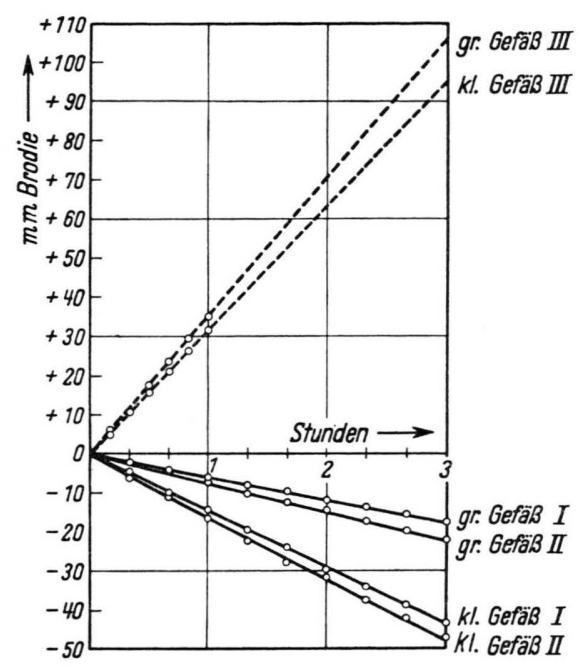

Abb. 1. Manometrie der weißen Blutzellen in Serum für je $6,44 \mathrm{mg}$ Zellen (Trockengewicht). $38^{\circ}$. $5 \% \mathrm{CO}_{2}$-Luft. Kurven I: Weiße Menschenblut-Zellen $Q_{O_{2}}=-3,3 ; Q_{\mathrm{CO}_{2}}=+3,3$; $Q_{\mathrm{M}} \mathrm{O}_{2}=0$. Kurven II : Weiße Hühnerblut-Zellen $Q_{0_{2}}=-2,8$; $Q_{\mathrm{CO}_{2}}=+2,1 ; \quad Q_{\mathrm{M}} \mathrm{O}_{2}=0$. Kurven III: Asciteskrebs-Zellen. $Q_{\mathrm{O}_{2}}-8 ; Q_{\mathrm{CO}_{2}}+33 ; Q_{\mathrm{M}} \mathrm{O}_{2}+25$. Kleines Gefäß V=16,10 $\mathrm{cm}^{3}$, $V_{\mathrm{F}}=7,35 \mathrm{~cm}^{3}$. Großes Gefäß $\mathrm{V}=23,34 \mathrm{~cm}^{3}, V_{\mathrm{F}}=7,35 \mathrm{~cm}^{3}$.

Ebenso wie der in Abb. 1 beschriebene Versuch, wurden 5 Versuche mit weißen Blutzellen des Menschen ausgeführt.

Die Tab. 1 zeigt, daß in keinem Fall die Gesamt$\mathrm{CO}_{2}$ größer war als der $\mathrm{O}_{2}$-Verbrauch, daß also Milchsäurebildung, falls eine solche vorhanden war, sehr klein war gegen den $\mathrm{O}_{2}$-Verbrauch. Wieviel von diesem Stoffwechsel von den Blutplättchen herrührte,

3 Positive Drucke erzeugt der Stoffwechsel immer dann, wenn der numerische Wert von $\gamma$ (Gesamt- $\left.\mathrm{CO}_{2} / \mathrm{O}_{2}\right)$ größer als $k_{\mathrm{CO}_{2}} / k_{\mathrm{O}_{2}}$ ist. 


\begin{tabular}{|c|c|c|c|c|}
\hline $\begin{array}{c}\text { Versuch } \\
\text { Nr. }\end{array}$ & $Q_{\mathrm{O}_{2}}$ & $\begin{array}{c}Q_{\mathrm{CO}_{2}} \\
(\text { Gesamt-CO }\end{array}$ & $Q_{\mathrm{M}}^{\mathrm{O}_{2}}$ & $Q_{\mathrm{M}}^{\text {Argon }}$ \\
\hline 1 & $-3,3$ & $+3,3$ & 0 & $+10,3$ \\
2 & $-3,1$ & $+3,2$ & 0 & $+12,6$ \\
3 & $-3,0$ & $+3,1$ & 0 & $+12,4$ \\
4 & $-2,8$ & $+2,6$ & 0 & +13 \\
5 & $-1,7$ & $+1,1$ & 0 & $+10,6$ \\
\hline
\end{tabular}

Tab. 1. Stoffwechsel von Leukocyten des Menschen.

wurde geprüft, indem die weißen Blutzellen bei höherem $g$ vollständig abzentrifugiert wurden und dann der Stoffwechsel in der darüber stehenden Flüssigkeit gemessen wurde, also der Stoffwechsel der Blutplättchen ohne Leukocyten. Es ergab sich, daß der Stoffwechsel nur einen kleinen Teil des Stoffwechsels der weißen Blutzellen ausmachte.

Vogelblut enthält nach den Angaben der Literatur keine Blutplättchen. Wurden die roten Blutzellen aus Hühnerplasma in gleicher Weise wie aus Menschenplasma durch fraktionierte Zentrifugierung abgetrennt, und wurde dann der Stoffwechsel der überstehenden Flüssigkeit, die also keine Blutplättchen enthielt, gemessen, so wurde gemäß Abb. 1 der gleiche Stoffwechsel wie für das Gemisch von Leukocyten und Blutplättchen des Menschenblutes erhalten. Zum Beispiel waren die Stoffwechselquotienten für Hühnerleukocyten im Hühnercitratplasma:

$$
Q_{\mathrm{O}_{2}}=-2,8 ; Q_{\mathrm{M}}^{\mathrm{O}_{2}}=0 ; Q_{\mathrm{M}}^{\mathrm{Argon}}=+6,7 .
$$

\title{
Atmung und Glykolyse der Rattenleber während der Behandlung mit 4-Dimethylaminoazobenzol*
}

\author{
Von H. Druckrey, F. Bresciani ** und H. Schneider \\ Aus dem Laboratorium (Leiter: Prof. Dr. Druckrey) der Chirurg. Universitätsklinik \\ (Direktor: Prof. Dr. H. Krauss) Freiburg i. Br. \\ (Z. Naturforschg. 13 b, 516-525 [1958]; eingegangen am 7. Mai 1958)
}

\begin{abstract}
1. At the example of the production of liver cancer in rats by feeding with 4-dimethyl-aminoazobenzene the problem is examined, whether the metabolism in the liver gradually turns into the cancer-metabolism or suddenly only then, when the carcinogenic degeneration is complete.

2. During the treatment the respiration of the liver evidently but not significantly decreases and is often higher with carcinomas than with the untreated controls.

3. During the treatment the aerobic and mainly the anaerobic glycolysis increase up to the threefold of the initial value. The strongest increase, however, takes place quickly and is detectable already in the beginning of the treatment.

4. After stop of the treatment the alterations completely disappear within 5 days and that also after the highest total doses 4-DAB. The earlier observed glycolysis can therefore not be considered as cancer metabolism, as more as it is inhibited by $\mathrm{n} / 250$ oxalate.

5 . The produced hepatomas show the characteristic tumor metabolism described by $\mathrm{W}_{\mathrm{ARBURG}}$. Now the alterations are irreversible. The change takes place abruptly.

6. The cancer-free parts of the same livers show after stop of the treatment a completely normal metabolism.

7. The described results support the view that the occurrence of the strong glycolysis is not the cause but the consequence of the carcinogenic degeneration.
\end{abstract}

Die Erzeugung von Hepatomen bei Ratten durch bestimmte Azo-Farbstoffe ermöglichte es, nun an einem großen homogenen Gewebe, nämlich der Leber, die wichtige Frage zu prüfen, ob der Stoffwechsel während der fortdauernden Behandlung unter stetiger Zunahme der Glykolyse allmählich in den WARBURGschen Krebsstoffwechsel ${ }^{1}$ übergeht

\footnotetext{
* Herrn Professor Dr. Dr. h. c. Boris Rajewsky zum 65. Geburtstag.

Die Untersuchungen wurden durch die Deutsche Forschung gemeins chaf t ermöglicht.

* Stipendiat des deutschen akademischen Austauschdienstes und des Consiglio nazionale delle Ricerche. Dauernde
}

oder vielmehr erst mit dem Auftreten von Tumoren sprunghaft erfolgt.

Die ersten Beobachtungen stammen von Hayashi und Tоміта ${ }^{2}$ (1937). Sie fanden in Fütterungsversuchen mit $o$-Amidoazotoluol, daß eine aerobe Glykolyse erst nach der Entwicklung von Hepatomen auftritt. Ihre Ergebnisse wurden von Nakatani,

Adresse: Istituto di Patologia generale dell'Universita di Napoli (Direktor: Prof. Dr. L. Califano).

1 O. Warburg, Der Stoffwechsel der Tumoren, Berlin 1926.

2 I. Hayashi u. T. Tomita, Gann, Japan. J. Cancer Res. 31, 232 [1937]. 